\title{
Freiheit, Gleichheit, Brüderlichkeit
}

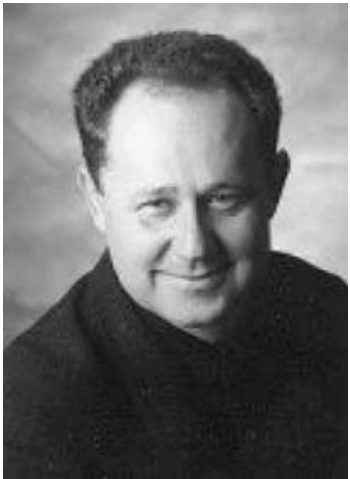

Prof. Dr. Manfred Wildner
Als François de Salignac de La Mothe-Fénelon, Erzbischof von Cambrai, in den Jahren 1693 und 1694 den Erziehungsroman "Die Abenteuer des Telemach“ für den Herzog von Burgund schrieb, geschah dies nicht ohne persönliche Gefährdung [1]. Da er bereits vom französischen Königshof wegen unbotmäßiger Publikationen unter scharfer Beobachtung stand, verzichtete er auf eine Publikation. Die Veröffentlichung, die sich erst 1699 im Ausland vollzog, ist der Untreue eines Kopisten zu verdanken, der diese Schrift anonym und wie man sagt ohne Wissen des Erzbischofs in Umlauf brachte - mit großem Erfolg.

Dass ihm 100 Jahre später die Begriffe Liberté, Égalité, Fraternité (Freiheit, Gleichheit, Brüderlichkeit) im Gefolge der französischen Revolution zugeschrieben werden würden, konnte Fénelon nicht ahnen. Diese Geschichte gibt jedoch ein beredtes Beispiel von der Macht der Ideen. Dass die staatliche Autorität letztlich ein Diener der Freiheit und des öffentlichen Wohls sein sollte, wie Fénelon im 18-ten Buch der „Abenteuer des Telemach" schreibt, ist eine dieser Ideen.

Ideen, wenn ihre Zeit gekommen ist, scheinen mehr als einmal geboren zu werden. Im Jahr 1776 wählte der Geheimorden der Illuminaten vom Ingolstädter Professor für Kirchenrecht Adam Weißhaupt gegründet - die Vervollkommnung und den Fortschritt der Menschheit in Freiheit, Gleichheit und Brüderlichkeit zum Motto. Der geheime Weisheitsbund wurde neun Jahre später verboten. Die Devise von Freiheit, Gleichheit und Brüderlichkeit wurde jedoch während der Französischen Revolution von Antoine-François Momoro wieder aufgegriffen und im Zusammenhang mit Veröffentlichungen zur Erklärung der Menschen- und Bürgerrechte (1792) popularisiert. Momoros ursprüngliche Formulierung lautete „Liberté, Egalité, Indivisibilité ou la mort“ (Freiheit, Gleichheit, [staatliche] Ungeteiltheit oder der Tod). Momoro wurde 2 Jahre später unter der Gulliotine in Paris enthauptet.

Die Sache mit der Freiheit scheint nicht so einfach zu sein. Der Weg bis hin zu einer Freiheitsstatue auf einer Liberty Island im New Yorker Hafen, als „Freiheit, die die Welt erleuchtet“ am 28. Oktober 1886 als Geschenk des Französischen Volkes an die Vereinigten Staaten eingeweiht, ist sicherlich lang und ganz sicher nicht ohne Opfer gewesen. Freiheit wurde insbesondere seit dieser Zeit zu einem Leitthema menschlicher Zivilisation und Kultur. „Freiheit, die ich meine“, singt Max von Schenkendorf als Liedtexter während der Befreiungskriege wiederum gegen die napoleonische Herrschaft. „Freiheit als Einsicht in die Notwendigkeit" formuliert Friedrich Engels mit Ver- weis auf Hegel: „Nicht in der geträumten Unabhängigkeit von den Naturgesetzen liegt die Freiheit, sondern in der Erkenntnis dieser Gesetze, und in der damit gegebenen Möglichkeit, sie planmäßig $z u$ bestimmten Zwecken wirken zu lassen" ([2]). „Freedom is just another word for nothing left to lose“, (Freiheit ist nur ein anderes Wort dafür, nichts mehr zu verlieren zu haben), singt Janis Joplin einige Tage vor ihrem Tod im Jahre 1970. Für Bobby McGee und wohl auch für sich selbst. Nicht zuletzt die Wissenschaft reklamiert ihre Freiheit, in Forschung und Lehre. Die Freiheit von Wissenschaft, Forschung und Lehre sind in Artikel 5, Abs. 3 GG als Grundrecht geschützt: „Kunst und Wissenschaft, Forschung und Lehre sind frei." Klammert man Kunst als eigenständigen Bereich aus, wird die Freiheit der Wissenschaft als Überbegriff durch die Begriffe von Forschung und Lehre als Forschungsfreiheit und Lehrfreiheit konkretisiert. Freiheit der Lehre ist eingängig doch was ist eigentlich wissenschaftliche Forschung? Nach der Definition des Bundesverfassungsgerichts ist wissenschaftliche Forschung ,jede Tätigkeit, die nach Inhalt und Form als ernsthafter planmäßiger Versuch zur Ermittlung der Wahrheit anzusehen ist" (BVerfGE 35, 79 - Hochschul-Urteil).

„Die Wissenschaft, sie ist und bleibt, was einer ab vom anderen schreibt. Und trotzdem ist, ganz unbestritten, sie immer weiter fortgeschritten“, dichtet Eugen Roth [3]. So ganz leicht möchte man die wissenschaftliche Kultur des Austausches nun doch nicht nehmen. Dass auch die Freiheit der Wissenschaft, wie jede Freiheit, verteidigt werden muss, z.B. wenn die Ernsthaftigkeit dieses Versuchs zur Ermittlung der Wahrheit aufgrund anders gerichteter Interessen gefährdet ist durch die planvolle Unterdrückung unliebiger Erkenntnis, durch wirtschaftlich dominiertes Gewinnstreben, durch Ich-bezogene Titelsüchtelei - ist ein beachtenswerter Aspekt (siehe auch [4]). Im Bereich der Publikationstätigkeit humanmedizinischer Fachzeitschriften wurden einheitliche Bewertungsmaßstäbe definiert [5]. Sie regeln u.a. die Publikationsethik hinsichtlich Sponsorenschaft, Autorenschaft und Verantwortlichkeit. Dass derartige Regeln aufgestellt werden, ist nicht nur ein freier Akt wissenschaftlicher Selbstverwaltung, sondern spiegelt auch das gegebene Konfliktpotential in diesem Bereich wieder.

Auch gut und detailliert ausformulierte Regeln müssen interpretiert, angewendet und umgesetzt werden. Von der deutschen Forschungsgemeinde (DFG) wurde auf Empfehlung der Kommission Selbstkontrolle in der Wissenschaft ein Ombudsman für die Wissenschaft eingerichtet. 
Diese Institution - ein Gremium - ist im Selbstverständnis eine Beratungs- und Vermittlungseinrichtung zu Fragen guter wissenschaftlicher Praxis. Sie kann von Wissenschaftlerinnen und Wissenschaftlern unmittelbar und unabhängig von der DFG angerufen werden. Grundlage ihrer Arbeit ist die DFG-Denkschrift "Sicherung der guten wissenschaftlichen Praxis“ [6].

So wie der Staat im Grundgesetz die Wissenschaftsfreiheit schützt und im Fénelonschen Verständnis der Sorge um Freiheit und Glück der Bürger auch schützen sollte - kann er sie nicht auch gefährden? Waren in der Vergangenheit nicht auch Einflussnahmen staatlicher Repräsentanten auf Prozesse der Wahrheitsfindung zu beobachten, bis in den besonders geschützten Bereich der Wissenschaftsfreiheit hinein? Welche Spannungsverhältnisse ergeben sich für staatliche und überstaatliche Behörden mit Kontrollfunktionen innerhalb von Macht und Interessensgefügen, die bisweilen hierarchisch klar zu benennen, oftmals nur schwer zu durchschauen sind?

Die Europäische Lebensmittelbehörde (European Food Safety Authority, EFSA) wurde im Jahr 2002 als unabhängige Körperschaft zum Risiko-Assessment im Lebensmittelbereich von der Europäischen Union gegründet. In einem frühen Grundsatzdokument wurde die fundamentale Bedeutung eines unabhängigen Amtes mit einer Rechtspersönlichkeit, welche von den Institutionen der Europäischen Union getrennt ist, herausgearbeitet [7]. Diese konkretisierte Gewaltenteilung verdient in ihrer Weitsicht und Einsicht der Selbstbeschränkung aufmerksame Beachtung. Wohl auch auf Grund gemachter Erfahrungen findet innerhalb der EFSA eine lebhafte Diskussion zur Unabhängigkeit wissenschaftlicher Stellungnahmen statt. Diese hat in ein weiteres Grundsatzdokument gemündet, welches sich u.a. mit der Unabhängigkeit und Wissenschaftlichkeit der internen Entscheidungsprozesse, mit Kernwerten der Behörde, mit diesbezüglichen organisatorischen Fragen, dem wissenschaftlichen Entscheidungsprozess als solchem, Aspekten der Qualitätssicherung und Organisationskultur und der Wahrung des öffentlichen Interesses befasst [8]. Das Dokument ist zur Kommentierung freigegeben, ihm sei gewünscht, dass es breit rezipiert und in angepasster Form auch in anderen Verwaltungszweigen verwendet wird.

Das Streben nach (Wissenschafts-)Freiheit verlangt tatkräftige Umsetzung, u. U. auch Kraft zu Widerstand und Kampf, bisweilen auch zum Opfer und zum Erdulden von Verfolgung. Dabei ist ja nicht Freiheit alleine gefordert: auch Gleichheit und Brüderlichkeit sind genannt. Während im historischen Kontext die Gleichheit der politischen Freiheiten, der Würde und des Wertes der Individuen gemeint war, ergibt sich unter dem Blickwinkel der Fragen von Krankheit und Gesundheit noch ein anderer Aspekt. Gleichheit betrifft hier auch die nie auszuschließende Verwundbarkeit allen menschlichen Lebens: vor dem Tod sind alle Menschen gleich. Doch halt, hinsichtlich des Eintrittszeitpunktes des Todes bzw. von Krankheit und hinsichtlich der Umstände im Vorfeld auf unserem Lebensweg hin zum Tode gibt es wohl doch erhebliche Unterschiede. Die sozialepidemiologische Forschung hat bedeutsame Anmerkungen zu machen, was die Chancen und Risiken bezüglich gesunder oder schlechter Gesundheit, Zugang zu Versorgungsleistungen im Falle von Krankheit und der Versorgung in den letzten Tagen und Wochen vor dem Tod betrifft. Zu diskutieren sind auch eine (Un-)Gleichheit der allgemeinen Lebensrisiken unserer „Risikogesellschaft“ (Ulrich Beck), Fragen der Umweltgerechtigkeit und sonstigen gruppenspezifischen Vulnerabilitäten.
Wissenschaftsbasiert, transparent und unabhängig engagieren sich auch die Autoren in diesem Heft: mit Beiträgen zum populationsbezogenen Mammographie-Screening in Deutschland und zu sozialen Ungleichheiten bei der Inanspruchnahme der onkologischen Rehabilitation, zur Auswirkung von Mindestmengen bei Perinatalzentren, zu Patientensicherheitsindikatoren der Arzneimitteltherapie und zur epidemiologischen Erfassung unerwünschter Ereignisse mit dem Harvard Medical Practice Design, zu einem „Aktionsbündnis gesunde Lebensstile und Lebenswelten“ und zu Bewegung und Ernährung an Oberfrankens Schulen im Rahmen einer ressourcenorientierten Gesundheitsförderung.

Letztlich ist das „Programm Freiheit“, das sich die modernen Gesellschaften gegeben haben, mit einer Gleichheit im doppelten Sinne von Gerechtigkeit und Verwundbarkeit und auch mit einer dadurch mit bedingten Notwendigkeit zur Solidarität (als geschlechtsneutrale Formulierung von „Brüderlichkeit“) eng verbunden. Wenn wir uns als verwundbare und letztlich sterbliche Wesen begriffen haben, wenn wir gegen die Unfreiheit ungerechter Verhältnisse rebellieren, wenn wir frei sein wollen von den Geißeln von Krankheit und unzeitgemäßen Todesfällen, sind wir auch zur Solidarität gerufen. So sind auch die Fragen zur solidarischen Finanzierung und zur Organisation eines hochmodernen Gesundheitsversorgungssystems nicht rein ökonomischer Natur, bezogen auf einen wichtigen Wirtschaftsfaktor, schon gar nicht ein Mittel zum Zweck partikulärerer Interessen und Interessensgruppen, sondern ein gemeinsames Unterfangen der Daseinsbewältigung im Dienst an der nur gemeinschaftlich zu schaffenden Wohlfahrt („Glück“) der Bürger. Auch eine „individualisierte“ Präzisionsmedizin wird neue und eher zunehmende Herausforderungen an Zusammenarbeit, Zusammenhalt und Mitspracherechten mit sich bringen [9]. Unter diesem Aspekt des Ausgesetzt-Seins menschlichen Lebens in einem langfristigen auch evolutionsbiologisch mitbestimmten Menschwerdungsprozess, an dem wir zunehmend nicht nur Erleidende, sondern auch in einer Art Risikogemeinschaft miteinander verbundene Mitwirkende und Mitschaffende sind, bekommt Momoros Formulierung eine aktualisierte Bedeutung: „Freiheit, Gleichheit, Brüderlichkeit - oder der Tod“.

\section{Literatur}

1 Fenelon F. Les aventures de Telemaque. URL http://www.gutenberg. org/files/30779/30779-h/30779-h.htm Zugriff am 30.01.2012

2 Karl Marx/ Friedrich Engels - Werke. (Karl) Dietz Verlag, Berlin. Band 20. Berlin/DDR. 1962. »Herrn Eugen Dührung's Umwälzung der Wissenschaft", S. 32-135. 1. Korrektur. Erstellt am 30.08.1999. Zitiert nach: http://www.mlwerke.de/me/me20/me20_032.htm , S. 105

3 Roth E. Tierleben. München: Hanser; 1988 Seite 6

4 Strech D, Koch K, Klemperer D. Offenlegen und dann? Wir brauchen eine rationale Diskussion zu einem angemessenen Umgang mit Interessenkonflikten. Gesundheitswesen 2011; 73: 271-272

5 International Committee of Medical Journal Editors. URL www.icmje. org Zugriff am 27.01.2012

6 Deutsche Forschungsgemeinschaft. Sicherung guter wissenschaftlicher Praxis. 1998;URL http://www.dfg.de/download/pdf/dfg_im_profil/ reden_stellungnahmen/download/empfehlung_wiss_praxis_0198.pdf Zugriff am 30.01.2012

7 Europäische Kommission. White paper on food safety (Kapitel 4). URL http://ec.europa.eu/dgs/health_consumer/library/pub/pub06_en.pdf Zugriff am 30.01.2012

8 European Food Safety Authority. A Policy on Independence and Scientific Decision-Making Processes of the European Food Safety Authority. URL http://www.efsa.europa.eu/en/consultations/call/110707b. pdf Zugriff am 20.01.2012

9 Mirnezami R, Nicholson J, Darzi A. Preparing for precision medicine. N Eng J Med 2012, doi: 10.1056/NEJMp1114866 